\title{
Visual inertia of rotating 3-D objects
}

\author{
YANG JLANG, ALLAN J. PANTLE, and LEONARD S. MARK \\ Miami University, Oxford, Ohio
}

\begin{abstract}
Five experiments were designed to determine whether a rotating, transparent 3-D cloud of dots (simulated sphere) could influence the perceived direction of rotation of a subsequent sphere. Experiment 1 established conditions under which the direction of rotation of a virtual sphere was perceived unambiguously. When a near-far luminance difference and perspective depth cues were present, observers consistently saw the sphere rotate in the intended direction. In Experiment 2, a near-far luminance difference was used to create an unambiguous rotation sequence that was followed by a directionally ambiguous rotation sequence that lacked both the near-far luminance cue and the perspective cue. Observers consistently saw the second sequence as rotating in the same direction as the first, indicating the presence of 3-D visual inertia. Experiment 3 showed that 3-D visual inertia was sufficiently powerful to bias the perceived direction of a rotation sequence made unambiguous by a near-far luminance cue. Experiment 5 showed that 3-D visual inertia could be obtained using an occlusion depth cue to create an unambiguous inertia-inducing sequence. Finally, Experiments 2, 4, and 5 all revealed a fast-decay phase of inertia that lasted for approximately $800 \mathrm{msec}$, followed by an asymptotic phase that lasted for periods as long as 1,600 msec. The implications of these findings are examined with respect to motion mechanisms of 3-D visual inertia.
\end{abstract}

In the 1950s a number of psychologists started to investigate how motion in 2-D displays could result in the perception of depth and 3-D shape (J. J. Gibson, 1957; J. J. Gibson \& E. J. Gibson, 1957; Johansson, 1950; Wallach \& O'Connell, 1953). Wallach and O'Connell demonstrated that the processing of a dynamic, 2-D image over time could lead to the perception of a 3-D form-what they referred to as the "kinetic depth effect" (KDE). In their classic experiments, they rotated rigid 3-D shapes behind a transparent screen. Observers on the other side of the screen perceived the 2-D shadows as a rigid 3-D object under rotation. In the absence of motion, however, the 3-D percept disappeared. Wallach and O'Connell's experiments clearly showed that dynamic 2 -D visual displays are sufficient to give the impression of the 3-D form of an object. Similarly, Johansson (1978) showed that a static image of a person or object may be difficult to identify, but becomes unambiguous when it moves. Motion can be critical for apprehending 3-D structure.

The recovery of 3-D structure from motion (SFM) has been investigated extensively by many researchers. Studies on SFM have examined perceptual judgments of rigidity and coherence of 3-D objects (Braunstein, 1962; Braunstein \& Andersen, 1984; Petersik, 1979, 1980; Todd, 1982; Todd \& Bressan, 1990), objective 3-D direction of rotation (Braunstein, 1962; Petersik, 1979), perceived depth of 3-D figures (Petersik, 1979; Dosher, Landy, \& Sperling, 1989), perceived 3-D curvature or shape (Johansson,

This paper is based in part on Y.J.'s PhD dissertation. This research was supported by grants from Sigma Xi and the Miami University Committee on Faculty Research. Correspondence should be addressed to A. J. Pantle, Department of Psychology, Miami University, Oxford, OH 45056 (e-mail: pantleaj@muohio.edu)
1978; Lappin \& Fuqua, 1983; Todd, 1984, 1985; Todd \& Norman, 1991), and 3-D transformations under minimal conditions (Braunstein, Hoffman, \& Pollick, 1990; Lappin, Doner, \& Kottas, 1980). Two successive frames have been shown to be sufficient to recover a SFM percept (Dosher et al., 1989; Todd, 1985). Most work on SFM has focused on the initial perception of SFM-that is, the relationship among various cues for the perception of 3-D structure and how those cues are combined.

In contrast, the present study examined the stability of the initial perceptual organization over time and its influence on the perception of later 3-D object motion. Our investigation of SFM focused on the history of stimulation in order to reveal some of the dynamic characteristics of 3-D motion perception. Toward this aim, we constructed two types of displays. The first was an unambiguous display that could be reliably perceived by observers as a stable percept. The perception of this unambiguous display was likely to persist and affect perception at a later time. The second type of display consisted of perceptually ambiguous stimuli. Such stimuli can be perceived in more than one way by different observers or by the same observer at different times. The ambiguous percept was thought to be unstable and prone to be biased by a previous robust percept.

\section{Ambiguous 3-D Motion Displays}

The ambiguous displays were parallel projections of rotating transparent spheres containing 30 randomly placed luminous dots. Whereas a single picture of this virtual sphere does not provide unambiguous information about depth, a sphere rotating slightly between the frames of a sequence usually results in a vivid perception of a 3-D rotating object. Even though depth can be recovered under parallel projection, the dots at different depths do not provide unambigu- 
ous information about depth order because projected speeds of the dots on the near and far sides of the sphere are equivalent. When the dots that appear to be closer to an observer move from right to left and the dots that appear to be farther move from left to right simultaneously, the transparent sphere rotates in a clockwise (CW) direction (and vice versa). For the sake of clarity of description, rotation directions are described with respect to the $y$-axis even though an observer's line of regard was coincident with the $z$-axis. Since there is a $50 \%$ chance that an observer perceives the leftward (rightward) moving dots as closer, the probability that an observer perceives a $\mathrm{CW}$ $(\mathrm{CCW})$ rotation is $50 \%$ (chance) under parallel projection (Braunstein, 1962; Petersik, 1979).

\section{Depth Cues: Disambiguating Depth Motion in 3-D}

To create a constant and stable perception of depth order that can disambiguate the direction of a 3-D rotation, additional depth cues must be added to SFM displays. Human psychophysical data have shown that our visual system combines different depth cues to arrive at a unified percept (Dosher, Sperling, \& Wurst, 1986; Landy, Maloney, Johnston, \& Young, 1995; Maloney \& Landy, 1989). The present experiments attempted to use three depth cuesperspective, luminance difference, and occlusion - in order to disambiguate the depth order and thereby the direction of a pattern of 3-D rotating dots.

Perspective cue: Polar projection. Under polar projection, the size of a projected image varies as the viewing distance changes. Consequently, perspective information makes the projected speeds of dots that are close to the observer appear to be greater than the speeds of dots that are far from the observer. As a result, perspective information may be able to disambiguate perceived depth, provided that the projection point is sufficiently close to the rotating object (Braunstein, 1976; J. J. Gibson, 1957; Johansson, 1974; Petersik, 1979). For this reason, polar projection was incorporated into some of the present dynamic displays in an attempt to obtain an unambiguous direction of 3-D rotation. Yet, a pilot study for the present experiments (Jiang, Pantle, \& Mark, 1994) and other research (Dosher et al., 1986) have shown that perspective cues may not always be sufficient to specify depth order unambiguously. To maximize the certainty of a perceived rotation direction, other depth cues were also included in the unambiguous displays used in this investigation.

Near-far luminance difference. A near-far luminance difference (contrast) in depth, also known as "aerial perspective," is a depth cue in which far light objects appear less bright than near objects. This phenomenon results from the scattering of light by particles in the atmosphere. J. J. Gibson (1979) considered the luminance reduction of bright objects with distance as an invariant (O'Shea, Blackburn, \& Ono, 1994). Dosher et al. (1986) used a luminance difference to disambiguate the perception of a rotating Necker cube. They named this depth cue "proximityluminance covariance" (PLC). In addition, they suggested that linear perspective was a weaker depth cue than PLC. Only a few studies have examined luminance difference as a depth cue, with most focusing on static depth perception (see O'Shea et al., 1994, for a review). In this study, PLC was created by assigning different luminance values to dots in the near and far halves of a transparent sphere (Experiments 1-4).

Occlusion. In static occlusion or interposition, a stationary object is blocked from view by a stationary surface that is closer to the observer. The interposition of objects may be a cue for relative distance. Kinetic occlusion occurs when a moving object is progressively covered and uncovered by an occluding surface (J. J. Gibson, Kaplan, Reynolds, \& Wheeler, 1969). This cue has been shown to help an observer separate dots into near and far depth planes (Braunstein, Andersen, \& Riefer, 1982). In the present study (Experiment 5), occlusion was added to the 3-D dynamic display to disambiguate the depth order of the rotating dots. Occlusion was created by inserting an opaque (wide or narrow) bar in the center of a simulated transparent sphere. When luminous dots rotated behind the bar, they went out of sight.

\section{3-D Visual Inertia}

The present experiments examined whether the perception of the direction of rotation of a simulated 3-D sphere could be biased by a previously perceived unambiguous rotation of the sphere. This bias or influence is referred to as "3-D visual inertia." Each experiment used dynamic displays containing dots rotating in 3-D. Depth cues were manipulated to produce either an unambiguous or ambiguous percept. The observer's task was to judge the direction of two successive rotations. Two types of tests were used to investigate the existence and temporal course of 3-D visual inertia. (1) In the first type of test, the first rotation sequence always contained additional depth cues so that the depth order of dots was unambiguous. Thus, the first rotation was directionally unambiguous. The second rotation sequence did not contain additional depth cues. By itself, this sequence was ambiguous for depth order and rotation direction; 3-D inertia occurred if the second ambiguous rotation was seen to be in the same direction as the first unambiguous rotation at a rate greater than chance. (2) The second type of test presented two successive unambiguous rotations in opposite directions. Observers should see the reversal of the perceived direction of the two rotations if each rotation was perceived independently. Failure to perceive a direction reversal would define a second type of 3-D visual inertia. In both types of displays, perceived direction of rotation was established with the assignment of depth order. Any 3-D visual inertia would be expressed as a resistance to change of direction when a rotation sequence was perturbed.

In summary, the present study investigated whether 3-D visual inertia exists and can influence judgments of the direction of rotation of a 3-D transparent sphere, as well as the time course of any visual inertia effects. Experiment 1 verified that the direction of a single 3-D rotation containing perspective and luminance depth cues was unambiguous. 


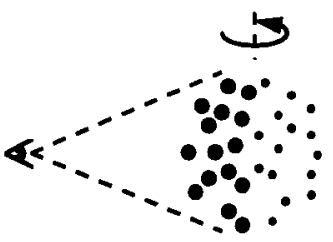

(A)

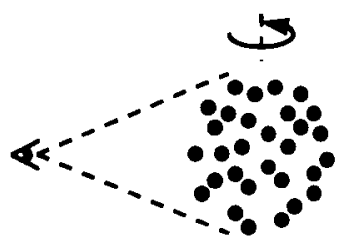

(C)

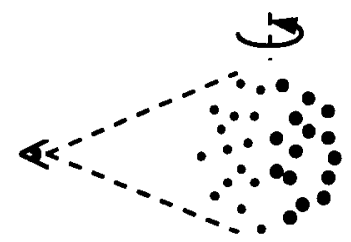

(B)

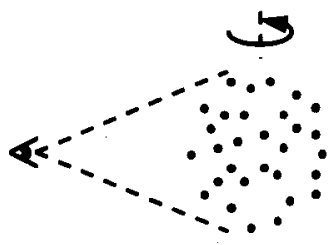

(D)
Figure 1. A schematic of the four types of displays used in Experiment 1, with large dots representing bright dots in the actual displays and small dots representing dim dots in the actual displays. Random dots conformed to one of four luminance patterns under polar and parallel projection. (A) Dots in the near half of the sphere were brighter than those in the far half of the sphere. (B) Dots in the near half of a sphere were dimmer than those in the far half. (C) Every dot had an equally high intensity (all bright dots). (D) Every dot had an equally low intensity (all dim dots). For each condition, there were equal numbers of clockwise and counterclockwise rotations.

\section{EXPERIMENT 1 \\ The Effects of Perspective and Luminance Depth Cues on Perceived Direction of a Single-Rotation Sequence}

Previous research has shown that the direction of a rotating transparent sphere is ambiguous under parallel projection. Observers' reports of the direction of rotation were, on average, equally divided between $\mathrm{CW}$ and $\mathrm{CCW}$ (Braunstein, 1976; Petersik, 1979). Since directional ambiguity is due to reversible near-far relationships of depth order under parallel projection, additional depth cues were expected to help disambiguate the perceived direction of rotation. Experiment 1 examined the effects of four different luminance displays combined with two types of projection on the perceived direction of a rotating sphere. The first experiment was designed to reveal any potential effects of the two depth cues (perspective and near-far luminance difference) on judgments of rotation direction. We were not attempting to produce the best SFM stimuli or to establish unequivocally the relative efficacy of the two cues. Rather, we were trying to find unambiguous stimuli that could serve as biasing stimuli in our later experiments, and yet not produce significant motion adaptation.

\section{Method}

Observers. Ten undergraduates from Miami University participated in the experiment to fulfill a course requirement. They were unaware of the purpose of the experiment. All observers had normal or corrected $20 / 20$ acuity.
Displays. The stimuli used in Experiment 1 were generated and displayed on a VGA color monitor (IBM Model 1812) driven by an IBM PC (PS/2 Model 30) computer. Each apparent motion sequence contained seven picture frames $(320 \times 200$ resolution) that were generated off-line before each trial and stored in the graphics memory of the computer. For a trial, the display of the seven frames in a rotation sequence was locked to the vertical sync signal of the monitor, and all frame durations were multiples of the monitor refresh rate $(70 \mathrm{~Hz})$. Each of the seven frames was presented for $100 \mathrm{msec}(7$ screen refreshes), except for the first frame, which lasted $2,857 \mathrm{msec}$ (200 screen refreshes). The frame rate was kept low so that the overall sequence duration was not too short, but high enough to create the appearance of smooth motion.

The display was a projection of a transparent sphere rotating around its vertical axis. Each successive frame advanced the rotation by $15^{\circ}$. Each frame contained 30 luminous dots. The dots were randomly placed within the volume of the simulated sphere, so that dot density was randomly distributed within the sphere. The $x$ (the horizontal axis), $y$ (the vertical axis), and $z$ (the depth axis) values of each dot were chosen randomly from all possible positions within a cube. The dots with values of $x^{2}+y^{2}+z^{2}>$ radius $^{2}$ (radius $=$ $5.75 \mathrm{~cm}$ ) were rejected. Each illuminated dot was green and seen against the dim background of the computer screen. The number of frames and the number of dots/frame were deliberately kept small to minimize motion adaptation. A cardboard surround with a circular hole of $7-\mathrm{cm}$ radius was placed in front of the computer screen. The simulated sphere was centered in the aperture. The computer screen and the cardboard surround had a luminance of $0.01 \mathrm{fLs}$. The rest of the room was dimly lit.

At the viewing distance of $200 \mathrm{~cm}$, the diameter of each projected sphere subtended roughly $3.3^{\circ}$ of visual angle. The simulated viewing distance for the polar projection was 1.5 sphere radii $(17.3 \mathrm{~cm})$ that contained strong perspective information. Although the actual and simulated viewing distances did not match, no shape distortion was visible when the perceived direction matched the simulated direction, as happened most often (see results section). Observers viewed the displays binocularly.

Experimental conditions. Figure 1 shows the four luminance conditions that were presented under either polar or parallel projection: (A) The dots' luminance in the near half of a sphere was higher than that in the far half of the sphere (18.4 vs. $5.1 \mathrm{fLs})$; (B) the luminance of dots in the near half of a sphere was lower than that in the far half of the sphere; (C) every dot had an equally high luminance (18.4 fLs); or (D) every dot had an equally low luminance (5.1 fLs). For each condition, there were equal numbers of simulated $\mathrm{CW}$ and $\mathrm{CCW}$ rotations. Under polar projection, the perspective information was always consistent with the simulated direction of rotation. Sixteen different displays ( 4 luminances $\times 2$ projections $\times 2$ directions of rotation) were presented 160 times for each observer in a random order. For each trial, the computer picked 30 new dots at random.

Procedure. After observers were adapted to the dark room for a minimum of $10 \mathrm{~min}$, they were given task instructions and several practice trials. The observers were instructed to look globally at all of the rotating dots and maintain a steady fixation toward the center of the display. Their task was to judge the direction in which the transparent sphere appeared to rotate. The experimenter recorded the observer's verbal response after each trial. No feedback was given during the experiment. The interval between trials was $8-10 \mathrm{sec}$.

\section{Results and Discussion}

This experiment examined the effects of a near-far luminance difference and a perspective cue on the perceived direction of a rotating sphere. For each individual, the responses were tabulated as the percentage of reports of a $\mathrm{CW}$ or a CCW rotation. The perceived direction of rotation was compared with the simulated rotation direction. ${ }^{1}$ 


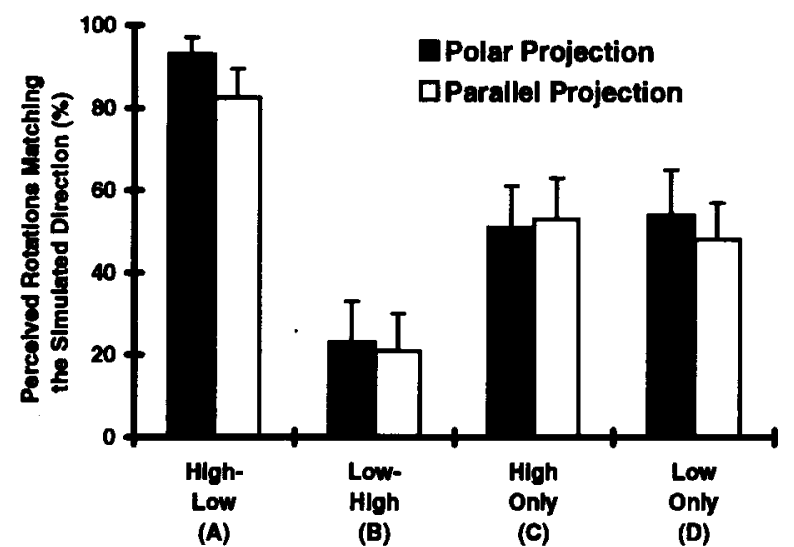

Luminance Pattern

Figure 2. The results of Experiment 1. The mean percentage of correct rotation judgments as a function of depth cues. High-low refers to display type $A$ in Figure 1; low-high refers to display type B; high only refers to display type C; and low only refers to display type $D$. Perceived direction of rotation was consistent with seeing the bright dots on the front half of the sphere. The perceived rotation direction was ambiguous without a luminance difference.

For each of the eight conditions, the percentage of trials for which each individual's perceived direction of rotation matched the simulated rotation direction (i.e., CCW responses to $\mathrm{CCW}$ stimuli were combined with $\mathrm{CW}$ responses to $\mathrm{CW}$ stimuli) was averaged across subjects to obtain a group mean percentage. Figure 2 shows the mean percentages and standard errors.

When there was no luminance difference in the displays (Sequence $\mathrm{C}$ or D), an observer's ability to judge the simulated direction of rotation was about chance: $52 \%(S E M=$ $10.4 \%)$ for high-luminance dots and $57 \%(S E M=11.7 \%)$ for low-luminance dots under polar projection, and $52 \%$ $(S E M=10.4 \%)$ for high-luminance dots and $48 \%(S E M=$ 9.5\%) for low-luminance dots under parallel projection. When the luminance of a dot in the near half of a sphere was higher than that in the far half of the sphere (Sequence A in Figure 1), the mean percentages (SEMs) of the perceived rotation directions matching simulated directions were $93 \%(S E M=3.5 \%)$ under polar projection and $83 \%$ ( $S E M=7.3 \%$ ) under parallel projection. That is, judgments of the direction of rotation were highly accurate when the luminance cue was in agreement with the perspective cue and the simulated rotation direction. When the luminance of the dots in the near half of a sphere was lower than that in the far half of the sphere (Sequence B in Figure 1$)$, only $25 \%(S E M=8.9 \%)$ under polar projection and $22 \%(S E M=8.9 \%)$ of the responses under parallel projection matched the simulated direction of rotation. That is, perceived direction was not accurate when the luminance cue was in conflict with perspective information and the simulated direction. Regardless of whether the judgments were accurate or inaccurate, in both conditions that contained luminance differences, the perceived di- rection of rotation was unambiguously specified by the luminance cue.

The direction discrimination performance obtained in this experiment under polar projection (without a luminance difference) was considerably worse than that reported in previous studies (Braunstein, 1976; Petersik, 1979). However, as noted earlier, our rotation sequences were brief (six motion steps in $42 \mathrm{msec}$ ) and contained only 30 dots. Also, the actual viewing distance did not correspond to the simulated viewing distance. The results indicate that perspective information, when present in our displays, was a weak depth cue; luminance as a depth cue overrode the perspective cue in determining rotation direction. This result is consistent with the finding of Dosher et al. (1986) that PLC was a stronger dynamic depth cue than linear perspective.

The conditions in Experiment 1 produced perceptually unambiguous and ambiguous displays of 3-D apparent rotation. The condition of near-high, far-low luminance dots in a sphere under polar projection (Sequence A) created a perceptually unambiguous sequence. Without a luminance difference in a pattern, an observer's perception of direction was ambiguous (Sequence $C$ or $D$ ). These results provided baseline conditions for examining 3-D visual inertia in Experiment 2.

\section{EXPERIMENT 2 \\ Visual Inertia Tested With Ambiguous Rotation Sequences}

Experiment 2 attempted to determine whether 3-D visual inertia exists, and if so, to identify some of its spatial and temporal properties. In contrast to the procedure used in the simultaneous spatial priming studies of Gillam (1972) and Eby, Loomis, and Solomon (1989), here we asked in what specific way, if any, the viewing of a sphere rotating in one direction affects the perception of a subsequent ambiguous rotation. In this experiment, a fourframe unambiguous rotation sequence was presented and followed by an ambiguous three-frame sequence. Also, 3$D$ visual inertia was defined as any influence of the first rotation sequence on the perceived direction of rotation of the second sequence.

In the present experiment, the temporal pause between the two rotation sequences was varied in order to determine the duration of any 3-D inertia. Also, we asked whether 3-D visual inertia generated by a sphere of one size would extend to a larger sphere.

\section{Method}

Observers. Eleven undergraduate students at Miami University served as observers in this experiment. They had normal or corrected 20/20 acuity and had not participated in Experiment 1.

Displays. As in Experiment 1, the simulated 3-D spheres were depicted by 30 dots randomly distributed in a spherical volume rotating either $\mathrm{CW}$ or $\mathrm{CCW}$ around the vertical axis. For each trial, the display contained seven frames (Figure 3). Except for the first and fourth frames, each frame lasted for $100 \mathrm{msec}$. The duration of the first frame was $2,857 \mathrm{msec}$, and it allowed an observer to achieve fixa- 


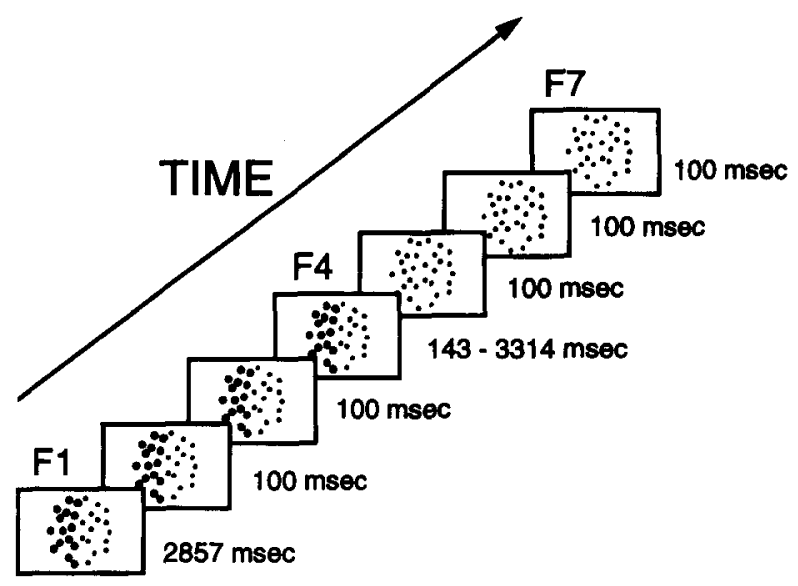

Figure 3. The seven frames used in the apparent motion sequences of Experiment 2. Each frame lasted 100 msec. An intersequence pause coincided with the duration of the fourth frame. A temporal pause of $143,543,829,1,657$, or 3,314 msec separated the two sequences.

tion before motion began. The duration of the fourth frame was lengthened in order to create two rotation sequences with a variable pause between them. We chose the pause over a blank interval because we wanted to stop motion, but yet have a stimulus available for maintaining fixation. The first (unambiguous) rotation sequence contained four frames and served as the priming stimulus, and the second (ambiguous) sequence contained three frames and served as the test stimulus. The positions of the dots for the second sequence were random and chosen independently of the positions in the first sequence.

The first rotation sequence (Sequence A in Experiment 1 reduced to four frames) contained near-high, far-low luminance dots consistent with the simulated direction of rotation and the perspective provided by polar projection. On the basis of the results of the first experiment, observers were expected to judge the first rotation sequence in accordance with its simulated direction of rotation. ${ }^{2}$ The next three frames (Sequence D in Experiment 1) contained only lowluminance dots under parallel projection. If viewed in isolation, Sequence $D$ should be ambiguous. Figure 4 illustrates the relative size of the displays used in the experiment. In half of the trials, a smaller version (diameter $=2.9^{\circ}$ ) of the sphere used as Sequence A in Experiment 1 was used as the priming sequence. In the other half of the trials, a sphere with a diameter of $4.5^{\circ}$ was used for the first sequence. Sequence D always contained the larger sphere. The duration of the fourth frame was $143,543,829,1,657$, or $3,314 \mathrm{msec}$.

For each trial, an observer saw an unambiguous rotation (one of the two sizes of Sequence A) followed by an ambiguous rotation (Sequence D). Observers judged the rotation direction of both sequences. Each individual viewed each of the 10 conditions 20 times ( 10 of the first rotations were $\mathrm{CW}$ and 10 were $\mathrm{CCW}$ ) in a random order. There were four possible responses for each trial: $\mathrm{CCW}-\mathrm{CCW}$, $\mathrm{CW}-\mathrm{CW}, \mathrm{CW}-\mathrm{CCW}$, or $\mathrm{CCW}-\mathrm{CW}$ directions. The time interval between trials in this and the remaining experiments was $8-10$ sec.

\section{Results and Discussion}

To test for the existence of 3-D visual inertia, an unambiguous rotation sequence was followed by an ambiguous sequence. Out of the four possible responses, judgments of CCW-CCW and CW-CW (same responses to each rotation) were tabulated and grouped into one category. ${ }^{3}$ The percentage of responses falling in the same category was averaged across the data for the 11 observers. The results (mean percentage and standard error) are plotted in Figure 5 for each of the 10 conditions. The solid and dashed lines in Figure 5 represent the data for the two sphere sizes.

The existence of visual inertia is indicated when both rotations for a trial were perceived to occur in the same direction on more than $50 \%$ of the trials. Figure 5 shows that visual inertia was present for short intersequence pauses. The percentage of same responses to Sequences A and D decreased over the interval from 143 to $829 \mathrm{msec}$ and reached an approximate asymptote of $50 \%$ around $800 \mathrm{msec}$. These results show that visual inertia persisted for roughly $800 \mathrm{msec}$. After $800 \mathrm{msec}$, the direction of the second rotation was perceived essentially independently of the first rotation. Figure 5 also indicates that there was no difference between the two size conditions; that is, the time course of 3-D inertia was virtually identical for the two sizes of the first priming sphere. The latter result shows that 3-D visual inertia extends across object size and also that it does not require priming and test stimuli to overlap completely in space. That complete spatial overlap was not required for our priming effects may indicate that they share some properties in common with the simultaneous spatial priming effects of Eby et al. (1989) and Gillam (1972).

\section{EXPERIMENT 3 \\ 3-D Inertia Tested With \\ Unambiguous Rotation Sequences}

Experiment 3 examined the strength of 3-D visual inertia using a different type of display. Unlike the displays used in Experiment 2, in which the second rotation direction was ambiguous, the present experiment used two successive unambiguous sequences depicting rotations in opposite directions (Sequences A and B from Experiment 1). In Experiment 2, it was found that an unambiguous rotation could influence the perceived rotation of an ambiguous sequence. In the present experiment, we asked whether the

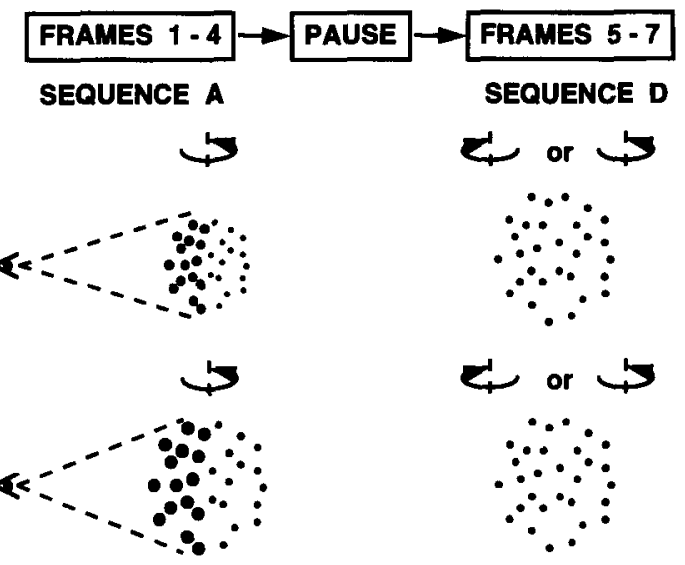

Figure 4. The displays used in Experiment 2. A large or small simulated sphere was followed by a large sphere with dim dots only. The first sequence contained perspective information, and the second sequence did not. 


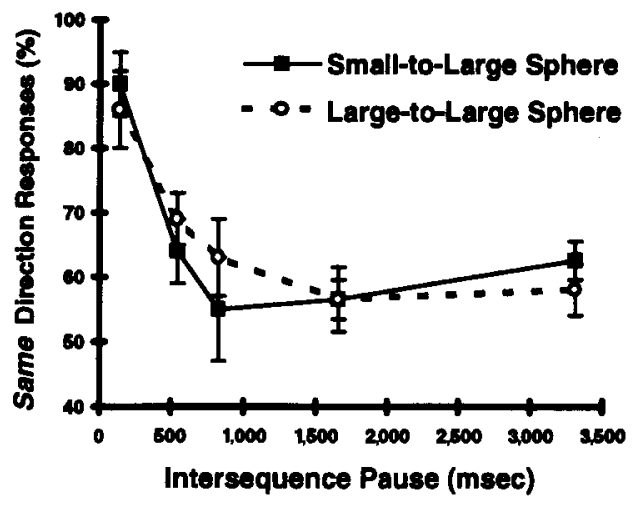

Figure 5. The results of Experiment 2. Percent same direction responses as a function of intersequence pause with sphere size as a parameter. With a short intersequence pause, 3-D visual inertia was found regardless of the size of the priming sequence. $A$ rapidly decaying visual inertia broke down when the intersequence pause exceeded 800 msec.

3-D inertia produced by an unambiguous sequence might not also be sufficiently powerful to bias the perception of an unambiguously rotating sphere.

Two unambiguous rotation sequences were created by the addition of a luminance difference cue. The first rotation contained near-high, far-low luminous dots. The dots in the second rotation followed the same trajectory as their counterparts in the first sequence; however, the luminance values assigned to the dots were switched (i.e., a near-low, far-high luminance pattern). In Experiment 1 it was shown that a change of near-far dot luminance (across trials) caused the perceived direction of rotation of a sphere to reverse. Therefore, in the present experiment it would be expected that the same luminance switch would cause observers to most often report a change in direction of rotation from the first to the second sequence, provided that the two sequences were perceived independently. Given the results of Experiment 1 and the assumption of independence, the expected probability of a perceived direction reversal in the present experiment was $.7 .{ }^{4}$ Reports that the second sequence reversed, with a probability less than .7 (i.e., its direction remained the same as for the first sequence, with a probability greater than .3), would demonstrate nonindependence, which we take as our definition of visual inertia with a display containing two successive unambiguous rotation sequences.

As a control for the ability to see direction reversals at all with our paradigm, we included conditions in Experiment 3 in which the simulated rotation direction was actually reversed between sequences, but dot luminance remained the same. For the control trials, the three frames of the second rotation sequence were simply the first three frames of the first rotation sequence displayed in reverse temporal order. Finally, the speed of rotation (frame duration) was varied in the experiment to examine the generality of any obtained 3-D inertia.

\section{Method}

Observers. Ten undergraduates with 20/20 acuity served as observers in the experiment. None had participated in the first two experiments.

Displays and experimental conditions. Each animation sequence contained a total of seven frames under polar projection. In the experimental conditions, the first four frames of the display were identical to the sequences of Experiment 2 that contained large spheres. These frames constituted the priming sequence. Dot luminance in the near half of the sphere was higher than that in the far half of the sphere. The dot luminance for the second three-frame sequence (test sequence) was reversed. For the control conditions, rotation direction, but not dot luminance, was switched between the two sequences. Figure 6 illustrates all the conditions in Experiment 3.

A sphere was made to rotate at either of two different apparent speeds by using frame durations of either 100 or $1,143 \mathrm{msec}$ under both the luminance-switching and direction-switching conditions. The time delay between the two rotation sequences was equivalent to the duration of the fourth frame. The apparent motion of the sequences with the 1,143-sec frame duration looked jerky. The long frame duration was intentionally chosen to see whether 3-D inertia would be manifest under conditions in which the apparent motion was not smooth. The same number of $\mathrm{CCW}$ and $\mathrm{CW}$ rotations were presented in each condition and for each rotation sequence in each condition. Each observer viewed 10 instances of each type of display in a random order, making a total of 80 trials. Again, observers were instructed to report the direction of the two rotations.

\section{Results and Discussion}

If the responses were $\mathrm{CW}-\mathrm{CW}$ or $\mathrm{CCW}-\mathrm{CCW}$, they were classified together in the same category. Percentages of responses in the same category were averaged across the 10 observers to obtain a group mean for each of the experimental and control conditions. The results are presented in Figure 7. The mean percentage of responses in the same category, along with standard errors, are shown for different combinations of rotation speed and luminance-switching and direction-switching conditions.

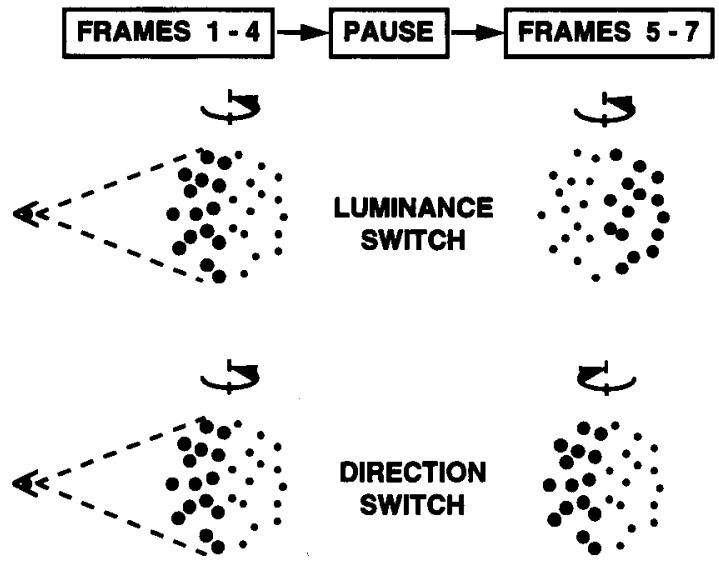

Figure 6. The displays used in Experiment 3. In the experimental condition (top), the luminance of the near and far dots was switched between the first and second rotation sequences. Rotation direction remained constant throughout the sequence. In the control condition (bottom), rotation direction was switched, but the near-far luminance difference was not. 


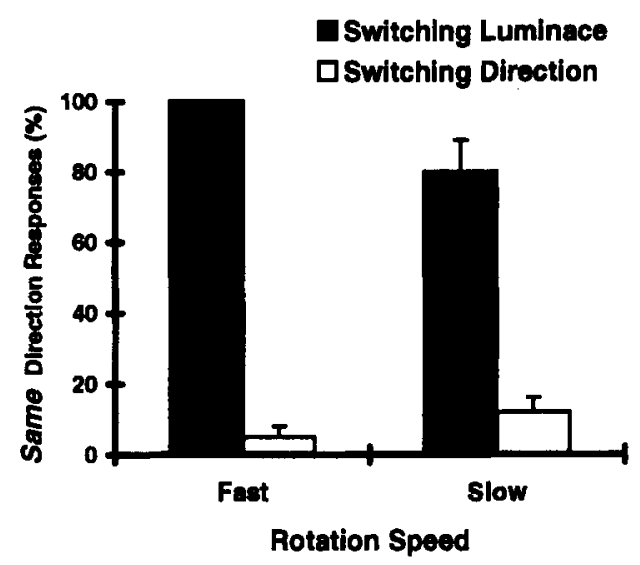

Figure 7. The results of Experiment 3. Percent same direction responses as a function of rotation speed. A change of dot luminance during a motion sequence did not reverse perceived direction of rotation for either a fast or a slow speed. A change of the simulated rotation direction reversed perceived rotation direction.

In the control condition, a switch of rotation direction resulted in a change in the perceived rotation direction. Only $2 \%$ of the observers' responses were in the same category with the fast rotation speed. Therefore, $98 \%$ were direction reversals $(\mathrm{CCW}-\mathrm{CW}$ or $\mathrm{CW}-\mathrm{CCW})$. Similarly for the slow speed, $87 \%$ were direction reversals, leaving $13 \%$ of the observers' responses in the same category. In the experimental condition (a switch of near-far dot luminance, but not direction of rotation), observers reported that both rotations were in the same direction for $100 \%$ of the trials with the fast rotation speed, and $81 \%$ with the slow rotation speed. The high percentages of responses falling in the same category provide strong evidence that the two rotation sequences in the experimental conditions were not perceived independently. The percentages far exceeded the .3 level expected under independence. Perceived direction of rotation of the second sequence was not readily controlled by near-far dot luminance, but was more directly related to the perceived direction of rotation of the first sequence. Apparently, 3-D visual inertia was able to override the effect of luminance difference as a depth cue.

In summary, Experiment 3 provides a converging measure for 3-D visual inertia. The inertia obtained with an unambiguous rotation following an unambiguous rotation complemented that found in Experiment 2, in which an unambiguous rotation followed an ambiguous rotation.

\section{EXPERIMENT 4 \\ Temporal Characteristics of 3-D Visual Inertia}

Experiments 2 and 3 demonstrated the existence of visual inertia for the perception of rotating stimuli. Further, Experiment 3 showed that the effect of visual inertia was sufficiently powerful to influence the perception of an unambiguous sequence. Experiment 4 extended Experiment 3 by examining the temporal characteristics of 3-D visual inertia with an unambiguous test sequence instead of an ambiguous test sequence (Experiment 2). The display used in the present experiment was identical to the fast rotation condition of Experiment 3 except that the display was altered by extending the duration of the last frame of the first rotation (fourth frame) or the first frame of the second rotation (fifth frame). Regardless of which frame was extended in time, the near-far dot luminance was switched on the fifth frame. The amount by which the duration of the fourth or fifth frame (intersequence pause) was extended provided a means for assessing the decay of visual inertia. By having the pause occur before or coincident with the luminance switch, it was possible to gain some insight into whether any processing time for the luminance switch contributed to 3-D visual inertia. Assume that the processing time is a significant part of any measured inertia. Then, when the fourth frame is extended, the pause would occur before the luminance switch on the fifth frame, and it would not be possible to process the luminance change before the second rotation begins. By delaying the luminance switch until the fifth frame, information about reversed depth order and reversed direction of rotation is delayed, and estimates of any inertia would be increased. On the other hand, when the fifth frame is extended, the luminance switch occurs before the pause. In this case, it is possible to process the luminance information about reversed depth order before the second rotation begins. Thus, when the second rotation sequence commences, information about reversed depth order and direction of rotation would be available immediately and would decrease estimates of the duration of inertia. Under an alternative assumption, the processing time for the near-far luminance cue would be short relative to any measured inertia. It should not matter whether the pause occurs before (fourth frame) or coincident with (fifth frame) the luminance switch.

\section{Method}

Observers. Ten undergraduate students at Miami University, who had not participated in the previous experiments, served as observers in this experiment. All observers had $20 / 20$ visual acuity.

Displays. The apparent rotation sequences used in this experiment were identical to those in Experiment 3, except that the duration of the fourth or fifth frame was varied. In different conditions, the duration was $200,400,600,800$, or $1,600 \mathrm{msec}$. The first four frames contained a near-high, far-low luminance dot pattern. Luminance switched between the front and the back of the simulated sphere for the next three frames (near-low, far-high luminance dots).

\section{Results and Discussion}

This experiment investigated the time course of 3-D visual inertia measured with an unambiguous test sequence. Again, responses were classified into two categories: perceived rotations in the same direction $(\mathrm{CCW}-\mathrm{CCW}$ or $\mathrm{CW}-\mathrm{CW}$ ) versus perceived rotations in opposite directions ( $\mathrm{CCW}-\mathrm{CW}$ or $\mathrm{CW}-\mathrm{CCW})$. The individual percentages and group means were obtained in the same way as in previous experiments. Figure 8 shows the average percentage of responses in the same category plotted as a function of 


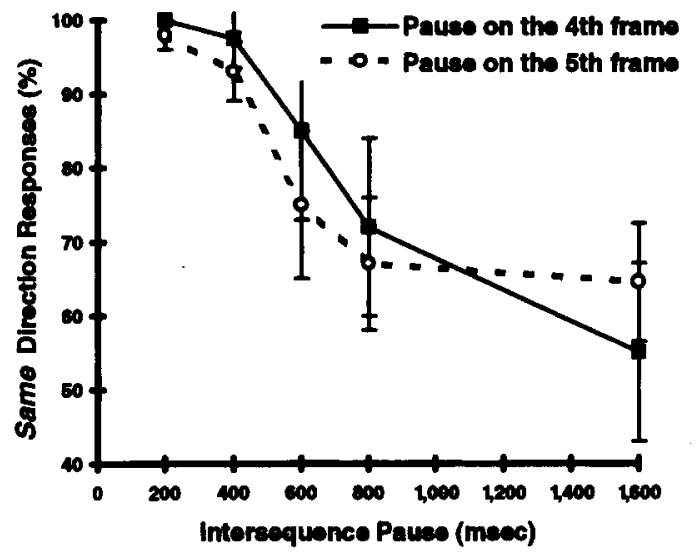

Figure 8. The results of Experiment 4. Percent same direction responses as a function of intersequence pause with pause location as a parameter. An intersequence pause was inserted on the last frame before the luminance switch (fourth frame of the sequence) or the first frame after the switch (fifth frame of the sequence). In either condition, visual inertia decreased as the duration of the pause increased.

the intersequence pause. The main effect of the duration of the intersequence pause was statistically significant $[F(4,9)=16.28, p<.001]$. Observers judged the second sequence to rotate in the same direction as the first nearly $100 \%$ of the time when the intersequence pause was $200 \mathrm{msec}$. The percentage of same responses decreased as intersequence pause increased, and it approached an asymptote of approximately $65 \%$ after $800 \mathrm{msec}$. Figure 8 also shows that the effect of the location of the intersequence pause did not have a significant effect on the decay function $[F(1,9)=0.75, p>.41]$.

The probability that an observer saw both sequences for a trial rotate in the same direction was well above .30 , the baseline criterion for independence of the two sequences computed from the empirical results of the first experiment. Thus, the results provide evidence for 3-D visual inertia for all intersequence pauses. Interestingly, the inertia declined rapidly for the first $800 \mathrm{msec}$, but never disappeared completely, remaining at the $65 \%$ asymptotic level for pauses as long as 1,600 msec. Factors that account for the initial time-dependent phase of inertia and for the time-independent constant (residual) level of inertia after $800 \mathrm{msec}$ may be different (see discussion section). The interaction between the temporal pause and the location of the frame at which the pause was placed was not statistically significant, but did approach significance $[F(4,36)=$ $2.57, p>.06]$, suggesting that the time to process the near-far luminance cue either did not extend the measured inertia or extended it by only a small amount.

\section{EXPERIMENT 5 Occlusion and 3-D Visual Inertia}

The generality of the 3-D visual inertia found in the previous experiments was established with displays that were marked by the presence or absence of perspective or near-far luminance cues to depth. In Experiment 2, the second rotation sequence did not contain perspective information, whereas perspective information was present in Experiments 3 and 4 . Likewise, a near-far luminance cue was present in the second rotation sequence of Experiments 3 and 4, but not in Experiment 2. Experiment 5 attempted to extend the generality of visual inertia by using still another depth cue, namely occlusion, to create unambiguous rotations. This attempt took the form of two questions: (1) Is 3-D inertia manifest across two unambiguous rotations when depth order given by occlusion is switched between the rotations? (2) If so, how do the temporal characteristics of 3-D inertia produced by occlusion compare with the inertia produced by the near-far luminance cue?

In this experiment, a simulated opaque bar was placed in the center of a rotating sphere defined by random dots. The bar occluded some dots and not others as they rotated around the bar. The occluded dots were seen as farther away than the nonoccluded ones. Thus, the occlusion separated the rotating dots into different depth planes. As determined in Experiment 1, when the depths of dots in the near and far half of a sphere were switched, the perceived direction of rotation also changed. In Experiments 3 and 4, a change in depth order between the two rotation sequences was accomplished by reversing the luminances of dots at near and far depths. In Experiment 5, the same effect was achieved by switching the occluded and nonoccluded dots whose positions overlapped the area of the bar.

\section{Method}

Observers. Ten undergraduate students with $20 / 20$ acuity served as observers in this experiment. None had participated in any of the previous experiments.

Displays. Figure 9 shows the display used in Experiment 5. The display was identical to that used in Experiments 3 and 4 with the following exceptions. The diameter of the sphere subtended $3.3^{\circ}$. Occluding bars of two different widths were placed in the center of the simulated spheres. Wide bars subtended a visual angle of $1.2^{\circ}$. Narrow bars subtended a visual angle of $0.6^{\circ}$. Each bar was taller than the sphere, extending to the edges of the aperture in the surround. The luminance of all dots was $18.5 \mathrm{fLs}$, and the luminance of the bar was $11.6 \mathrm{fLs}$. The fourth frame of a seven-frame display was extended in time to create a pause between two rotation sequences. Five intersequence pauses of $200,514,800,1,600$, or $3,200 \mathrm{msec}$ were used to examine the temporal characteristics of any visual inertia. Random dots occluded by an opaque bar in the first rotation sequence for a trial became visible in the second rotation sequence, and vice versa. The change of occlusion occurred with the fourth frame of the display. An observer's task was to judge the direction of the two rotations.

\section{Results and Discussion}

The reduction of individual data to group means was the same as in the earlier experiments. Figure 10 shows the mean percentages and the standard errors for responses in the same category as a function of the width of the occlusion bar and the duration of the intersequence pause. A $2 \times$ 5 within-subjects analysis of variance (ANOVA) showed that the width of the occluding bar significantly affected the 

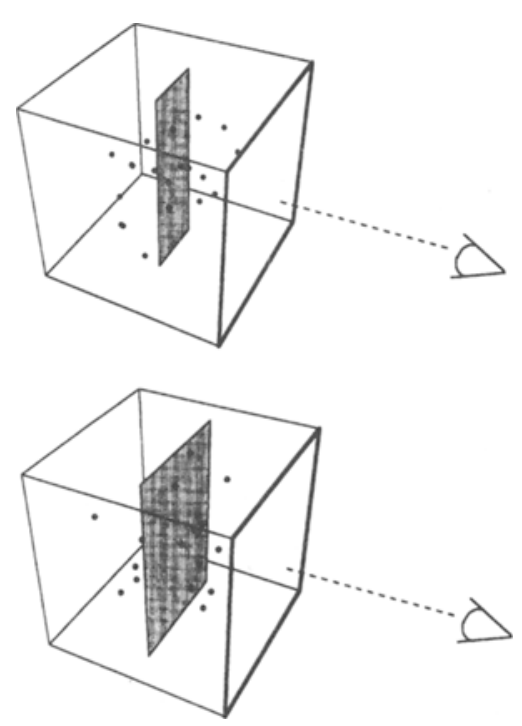

Figure 9. The displays of Experiment 5. An opaque bar was placed in the center of a simulated 3-D cloud of dots. In the figure, a cube was drawn enclosing the dots. The cube was drawn only for clarity and was not part of the actual display. The surface of this cube with the thick outline depicts a virtual window that faces the direction from which the observer viewed the cloud of dots circling the occlusion bar. All dots were visible to the observer except those occluded by the opaque bar. Two bar widths (wide and narrow) were used in the displays.

percentage of same responses $[F(1,9)=22.93, p<.001]$. A greater number of same responses was obtained with the narrow occluding bar than with the wide occluding bar.

Increasing the duration of the intersequence pause significantly reduced the percentage of same responses $[F(4,36)=27.52, p<.0001]$. The interaction between the width of the occluding bar and the duration of the intersequence pause was not significant $[F(4,36)=0.83, p>$ $.51]$. For both the wide- and narrow-bar conditions, the percentage of same responses declined rapidly during the first $800 \mathrm{msec}$, and it approached an asymptote of approximately $50 \%$ and $35 \%$ for the narrow- and wide-bar conditions, respectively. The time course of visual inertia with the occlusion cue used to specify direction of rotation was essentially the same as that obtained in Experiment 4 with the near-far luminance cue.

A breakdown of the responses to the first and second rotations for trials revealed that the direction of the first rotation was perceived correctly on $91 \%$ of the wide-bar trials, but only $81 \%$ of the time for narrow-bar trials. This is evidence that the wide bar provided more useful direction information than the narrow bar. Despite the seemingly stronger cue to depth order provided by the wide occlusion bar during the first rotation sequence, it did not give rise to more inertia. However, whenever the wide bar was present during the first rotation, it was also present during the second rotation. Consequently, any tendency for an observer to experience more inertia with the wide bar than with the narrow bar may have been counteracted by the ability of the wide bar, during the second rotation, to provide stronger information than the narrow bar that the sphere had reversed.

\section{GENERAL DISCUSSION}

The nonindependence of the direction of rotation of two successively presented spheres defined by random dots defines 3-D inertia. In the present study, 3-D inertia was obtained in two different paradigms, one in which an early unambiguous rotation biased the perceived direction of a later ambiguous rotation and another in which an early unambiguous rotation overrode the biased direction of a later unambiguous rotation. The 3-D inertia decayed over time and was size independent to the extent investigated. A fast-decaying component of the inertia was complete after approximately $800 \mathrm{msec}$ and remained essentially invariant across a variety of experimental conditions. An asymptotic (residual) level of inertia extended for time periods as long as $1,600 \mathrm{msec}$ and depended on factors that contributed to the degree of direction ambiguity of the rotation used to measure the inertia.

Local motion of luminance-defined stimuli is generally attributed to the activity of motion-sensitive cells. When an object moves in one direction, motion-sensitive cells preferring that direction are activated. This results in an imbalance among the impulse rates of the activated cells and cells sensitive to other directions. If the local directional imbalance persists, it can create translational 2-D visual inertia for subsequently viewed stimuli in the same depth plane (Pinkus \& Pantle, 1997).

Now consider leftward and rightward moving dots viewed simultaneously within the visual field. In this case, two groups of cells, each signaling opposite directions of motion, are equally activated. If the signals arising from the oppositely moving dots constrain the motion seen in only one depth plane, the lack of any directional imbalance

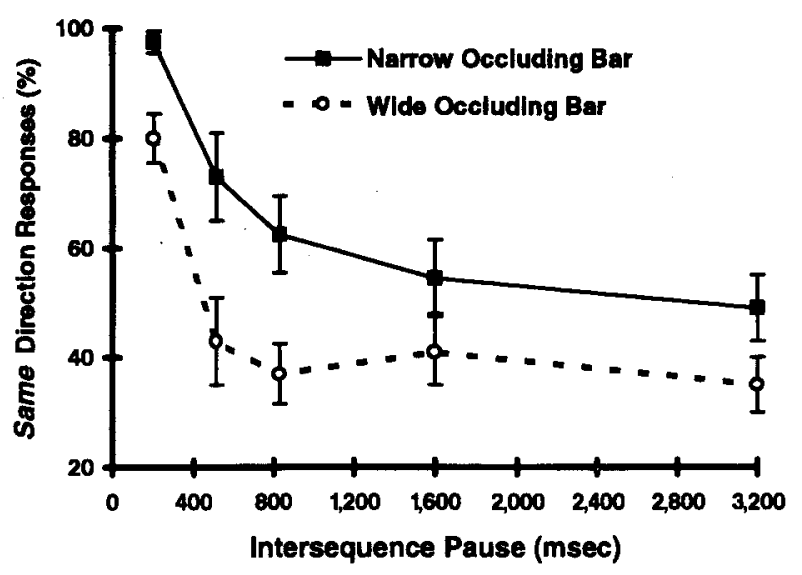

Figure 10. The results of Experiment 5. Percent same direction responses as a function of intersequence pause with width of the occlusion bar as a parameter; 3-D visual inertia produced by the occlusion decreased as a function of the intersequence pause. 
would presumably not result in any coherent global motion. This is what seems to have occurred in some paireddot displays investigated by Qian and Anderson (1994).

However, in many dynamic random-dot displays, dots moving in opposite directions are perceptually segregated into more than one depth plane. In transparent displays of this type, dots moving in one direction are arbitrarily assigned near depth values, whereas dots moving in the opposite direction are assigned far depth values (E. J. Gibson, J. J. Gibson, Smith, \& Flock, 1959). With variations of speed as well as direction, the linking of depth order and motion direction can give rise to a vivid percept of a rotating 3-D figure (Braunstein, 1976, pp. 85-94), as in any of the present rotation sequences. When the assignment of depth values to the direction of motion of dots is not consistent from one time to the next, the perceived direction of rotation of the 3-D figure will vary.

It may be that the 3-D visual inertia found in the present study was nothing more than the preservation of the linkages between individual 2-D-dot motions and assigned depth values during the transition from the first rotation sequence to the second in our paradigm. Consider, for example, the rotation sequences in Experiment 2. During the first rotation sequence on a trial, bright dots (say, those moving to the right) were assigned near depth values. The linkage between right and near would have constrained the 3-D direction of rotation to be CCW. During the second rotation sequence, the luminance difference between rightward- and leftward-moving dots disappeared. Therefore, the depth order of the dots was ambiguous, but the ambiguity could be resolved by tagging the dots with their 2-D motion direction and by noting at what depth those tagged dots were seen during the first rotation. Any tendency for the directionally tagged dots to inherit depth values from dots moving in the same direction during the first rotation would have constrained the direction of rotation during the second sequence to be the same as that during the first rotation. To continue the example above, rightwardmoving dots in the second sequence would have been matched to the rightward-moving dots during the first sequence, and they would have inherited their depth values from them, thereby maintaining the perceived $\mathrm{CCW}$ direction of rotation. In this view, 3-D visual inertia is a direct consequence of a tendency to preserve the couplings between 2-D direction signals and depth signals generated by moving dots. In Experiments 3-5, the tendency to preserve linked direction depth signals across sequences must have been stronger than the luminance and occlusion cues to depth order during the second rotation sequences. Otherwise, no 3-D motion inertia would have been manifest.

Our 3-D inertia and the basic assumption about the persistence of coupled 2-D direction and depth signals appears to have much in common with a priming phenomenon observed by Nawrot and Blake (1993) using a dynamic stereoscopic display and their model of the conjoint coding of binocular disparity and 2-D motion direction (Nawrot \& Blake, 1991b). In one of a series of experiments (Nawrot \& Blake, 1993), observers viewed a test display contain- ing dots randomly placed on the surface of a simulated globe. The globe was imaged with zero disparity and viewed under parallel projection as it rotated around a vertical axis. By itself the direction of rotation of the globe was ambiguous. A priming stimulus contained two sheets of dots moving in opposite directions, one sheet with dots of crossed disparity; and the other with dots of uncrossed disparity. Viewed alone, the priming stimulus appeared as two planes of oppositely moving dots, one near and one far relative to the point of fixation. When the priming and test stimuli were combined by presenting the priming stimulus $1 \mathrm{sec}$ before the globe and then temporally overlapping it for an additional $2.5 \mathrm{sec}$, the direction of rotation of the globe was disambiguated.

Although similar, our experiments differ from that of Nawrot and Blake (1993) in a number of ways. First, their priming and test stimuli overlapped temporally. Therefore, it is possible that their observed disambiguation occurred during the time the stimuli were temporally coincident, much like "noise" dots are captured by "signal" dots in a random-dot sphere with less than $100 \%$ coherence (Petersik, 1979). In our experiments, priming and test sequences were successive, and our 3-D inertia must have been the result of a temporally extended bias that was produced by the priming sequence and whose time course we could measure. Second, the cues used to produce the unambiguous priming stimuli were different in the two studies. Nawrot and Blake's (1993) priming stimulus was made unambiguous by retinal disparity, whereas ours was made unambiguous by a near-far luminance cue or occlusion. On the one hand, the cue difference may provide nothing more than a basis for generalizing priming effects across cues. On the other hand, our results may require a different kind of theoretical interpretation.

Nawrot and Blake (1989) borrowed the idea of cells tuned conjointly to 2-D motion direction and depth to explain their priming phenomenon from one of their earlier studies on the aftereffects of 3-D rotation. Extended exposure to a stimulus rotating unambiguously in one direction caused a subsequent stimulus to appear to rotate in the opposite direction. A similar finding had been reported earlier by Petersik (1984) and later by Ziegler and Dowling (1995). What was unique about Nawrot and Blake's (1989) study was the use of two control conditions. In one, they found that an adapting stimulus containing motion in only one depth plane was not sufficient to produce a 3-D motion aftereffect. When observers adapted to a single, flat (2-D) sheet of dots moving in one direction, the adaptation had no effect on the perception of a subsequent transparent rotating figure, regardless of the depth at which the sheet was made to appear due to retinal disparity. However, in a second control condition, they found that simultaneous adaptation to two planes of dots moving in opposite directions at two different depths did produce a 3-D rotation aftereffect. They suggested that the activation and adaptation of neural units selectively sensitive to motion in opposite directions in different depth planes was a necessary condition for obtaining the aftereffect. There is strong phys- 
iological evidence for visual neurons whose response is contingent on the correct combination of motion direction and retinal disparity (e.g., Roy, Komatsu, \& Wurtz, 1992).

Although it is possible to attribute our priming effects to the action of individual cells tuned conjointly to 2-D motion and depth, there are reasons why we are hesitant to do so. First, no one has yet reported the existence of cells tuned conjointly to 2-D motion and depth based on a nearfar luminance cue or occlusion. Second, evidence for rotation aftereffects induced with figures in which the depth order of dots is based on a near-far luminance cue or occlusion is nonexistent, negative, or weak. Nawrot and Blake (1991a) found no aftereffects with a near-far luminance cue during adaptation, whereas Ziegler and Dowling (1995) demonstrated some aftereffects, but those obtained by the latter researchers were very long-lasting, showing little decay over $60 \mathrm{sec}$, unlike other aftereffects. Third, in informal experiments with extended adaptation to our own priming stimuli with a near-far luminance cue, we have been unable to elicit reliable rotation aftereffects. No 3-D rotation aftereffects have yet been reported with occlusion used to specify depth order and rotation direction. Fourth, despite the fact that all the 3-D inertia curves of Experiments 2, 4, and 5 were characterized by the same initial rapid-decay phase up to approximately $800 \mathrm{msec}$, the asymptotic levels of the curves were different. The inertia curve for Experiment 2 reached a level that is consistent with the independent perception of rotation direction for the two sequences within a trial. The inertia curves for Experiment 4 remained above the level expected under the independence assumption for intersequence pauses as long as $1,600 \mathrm{msec}$, and the curves for the two bar width conditions in Experiment 5 were significantly different in height, but not in shape. The different behavior of the two phases of the inertia curves across and within experiments may mean that the phases depend on at least partially different underlying processes. The characteristics of the residual inertia are suggestive of top-down processes. It has been shown that attention can play a role in judgments of the direction of rotation of 3-D stimuli. Ambiguous figures can be stabilized by attentional strategies (e.g., Necker cube; see Shulman, 1994, for a review). More specifically, Shulman found that observers were able to control the direction of rotation of transparent spheres using a cognitive strategy in which the attended surfaces were given a front/ back default interpretation. Residual 3-D visual inertia and conscious attentional control of 3-D rotation may share common underlying mechanisms.

To summarize, although our experiments strongly suggest that the visual system attempts to preserve different 2-D directions of motion in different depth planes over time, we are hesitant to assume that either the fast-decay phase of the 3-D inertia or the residual inertia we observed was the result of the action of individual cells tuned conjointly to 2-D motion and a near-far luminance cue, or occlusion. Until more systematic research is available on 3-D motion priming with variety of depth order cues, it seems premature to propose a detailed mechanism by which depth order information from a prior rotation sequence combines with on-line depth order information from a present sequence. It is possible that the two sources of information establish separate incomplete depth maps on their own and that they are combined additively into one depth map at a later processing stage. Alternatively, some more highly interactive, nonlinear process may be suggested by future data.

\section{REFERENCES}

Braunstein, M. L. (1962). Depth perception in rotating dot patterns: Effects of numerosity and perspective. Journal of Experimental Psychology: Human Perception \& Performance, 64, 415-420.

BraunsteIN, M. L. (1976). Depth perception through motion. New York: Academic Press.

Braunstein, M. L., \& Andersen, G. J. (1984). Shape and depth perception from parallel projections of 3-D motion. Journal of Experimental Psychology: Human Perception \& Performance, 10, 749-760

Braunstein, M. L., Andersen, G. J., \& Riefer, D. M. (1982). The use of occlusion to resolve ambiguity in parallel projections. Perception \& Psychophysics, 31, 261-267.

Braunstein, M. L., Hoffman, D. D., \& Pollick, F. E. (1990). Discriminating rigid from nonrigid motion: Minimum points and views. Perception \& Psychophysics, 47, 205-214.

Dosher, B. A., LANDY, J. S., \& SPERLING, G. (1989). Kinetic depth effect and optic flow-I. 3D shape from fourier motion. Vision Research, 29, 1789-1813.

Dosher, B. A., Sperling, G., \& Wurst, S. A. (1986). Tradeoffs between stereopsis and proximity luminance covariance as determinants of perceived 3D structure. Vision Research, 26, 973-990.

EвY, D. W., Loomis, J. M., \& Solomon, E. M. (1989). Perceptual linkage of multiple objects rotating in depth. Perception, 18, 427-444.

Gibson, E. J., Gibson, J. J., Smith, O. W., \& Flock, H. R. (1959). Motion parallax as a determinant of perceived depth. Journal of Experimental Psychology, 58, 40-51.

GiBson, J. J. (1957). Optical motion and transformations as stimuli for visual perception. Psychological Review, 64, 288-295.

GiBson, J. J. (1979). The ecological approach to visual perception. Boston: Houghton Mifflin.

Gibson, J. J., \& Gibson, E. J. (1957). Continuous perspective transformations and the perception of rigid motion. Journal of Experimental Psychology, 54, 129-138.

Gibson, J. J., Kaplan, G. A., Reynolds, H. N., JR., \& Wheeler, K. (1969). The change from visible to invisible: A study of optical transitions. Perception \& Psychophysics, 5, 113-116.

Gillam, B. (1972). Perceived common rotary motion of ambiguous stimuli as a criterion of perceptual grouping. Perception \& Psychophysics, 11, 99-101.

JiAng, Y., PANTLE, A., \& Mark, L. (1994). Priming of apparent rotation of a 3-D sphere. ARVO Abstract. Investigative Ophthalmology \& Vision Science, 35, 127.

JoHaNSSON, G. (1950). Configurations in event perception. Uppsala, Sweden: Almkvist \& Wiksell.

JOHANSSON, G. (1974). Projective transformations as determining visual space perception. In R. B. McLeod \& H. L. Pick (Eds.), Perception: Essays in honor of J. J. Gibson (pp. 117-138). Ithaca, NY: Cornell University Press.

Johansson, G. (1978). Visual event perception. In R. Held, H. W. Leibowitz, \& H. L. Teuber (Eds.), Handbook of sensory physiology (pp. 675-711). Berlin: Springer-Verlag.

Landy, M. S., Maloney, L. T., Johnston, E. B., \& Young, M. (1995). Measurement and modeling of depth cue combination: In defense of weak fusion. Vision Research, 35, 389-412.

LAPPIN, J. S., Doner, J. F., \& KotTAS, B. L. (1980). Minimal conditions for the visual detection of structure and motion in three dimensions. Science, 209, 717-719.

LAPPIN, J. S., \& FUQUA, M. A. (1983). Accurate visual measurement of 3D moving patterns. Science, 221, 480-482. 
MALONEY, L. T., \& LANDY, M. S. (1989). A statistical framework for robust fusion of depth information. In W. A. Pearlman (Ed.), Visual communication and image processing IV: Proceedings of the SPIE, 1199, 1154-1163.

NAWROT, M., \& BLAKE, R. (1989). Neural integration of information specifying structure from stereopsis and motion. Science, 244, 716-718.

NAWrot, M., \& BlaKe, R. (1991a). The interplay between stereopsis and structure from motion. Perception \& Psychophysics, 49, 230-244.

NAWrot, M., \& BLAKE, R. (1991b). A neural network model of kinetic depth. Visual Neuroscience, 6, 219-227.

NAWROT, M., \& BLAKE, R. (1993). On the perceptual identity of dynamic stereopsis and kinetic depth. Vision Research, 33, 1561-1571.

O'Shea, R. P., BlackbuRN, S. G., \& ONo, H. (1994). Contrast as a depth cue. Vision Research, 34, 1595-1604.

Petersik, J. T. (1979). Three-dimensional object constancy: Coherence of a simulated rotating sphere in noise. Perception \& Psychophysics, 25, 328-335.

PETERSIK, J. T. (1980). The efforts of spatial and temporal factors on the perception of stroboscopic rotation simulations. Perception, 9, 271-283.

PETERSIK, J. T. (1984). A three-dimensional motion aftereffect produced by prolonged adaptation to a rotation simulation. Perception, 13, 489-497.

Pinkus, A., \& PANTLE, A. (1997). Probing visual motion signals with a priming paradigm. Vision Research, 37, 541-552.

QIAN, N., \& ANDERSON, R. A. (1994). Transparent motion perception as detection of unbalanced motion signals: II. Physiology. Journal of Neuroscience, 14, 7357-7366.

RoY, J.-P., Komatsu, H., \& Wurtz, R. H. (1992). Disparity sensitivity of neurons in monkey extrastriate area MST. Journal of Neuroscience, 12, 2478-2492.

Shulman, G. L. (1994). Cognitive control of rotation in depth. Visual Cognition, 1, 3-65.

ToDD, J. T. (1982). Visual information about rigid and nonrigid motion: A geometric analysis. Journal of Experimental Psychology: Human Perception \& Performance, 7, 795-810.

ToDD, J. T. (1984). The perception of three-dimensional structure from rigid and nonrigid motion. Perception \& Psychophysics, 36, 97-103.

ToDD, J. T. (1985). Perception of structure from motion: Is projective correspondence of moving elements a necessary condition? Journal of Experimental Psychology: Human Perception \& Performance, 11, 689-710.

TodD, J. T., \& Bressan, P. (1990). The perception of 3-dimensional affine structure from minimal apparent motion sequences. Perception \& Psychophysics, 48, 419-430.

TodD, J. T., \& Norman, J. F. (1991). The visual perception of smoothly curved surfaces from minimal apparent motion sequences. Perception \& Psychophysics, 50, 509-523.

Wallach, H., \& O'ConNell, D. N. (1953). The kinetic depth effect. Journal of Experimental Psychology, 45, 205-217.
ZIEGLER, L. R., \& Dowling, W. J. (1995). The hierarchical nature of perceiving direction of motion in depth from optic flow. Vision Research, 35, 1435-1446.

\section{NOTES}

1. We used correct direction of rotation with reference to world or object coordinates employed in programming and describing the spheres in 3-D space. In actuality, a rotation simulated under parallel projection has no correct direction of rotation because the sign of the $z$-axis coordinate is simply discarded when the 2-D image is generated. Therefore, the designation of correct direction of motion is arbitrary and was used only as a baseline for assessing responses without any directional information.

2. The 11 participants were $93 \%$ correct in judging the direction of the first unambiguous rotation with four frames. This level of accuracy was consistent with the results of Experiment 1, in which a single rotation was depicted by seven frames. For a directional discrimination, a rotation containing four frames was just as effective as a single rotation depicted by seven frames.

3. If the perceived direction of the first sequence did not match the simulated direction, $\mathrm{CCW}-\mathrm{CCW}$ and $\mathrm{CW}-\mathrm{CW}$ responses were still included in the same category for the data analysis reported. We also found that the pattern of results was not changed in any essential way by excluding such trials because they were infrequent, typically occurring on $5 \%-7 \%$ of the trials in all experiments.

4. If the perceived direction of the two rotation sequences was independent, the probability of perceiving a direction reversal $[P($ Reversal $)]$ could be predicted from Experiment 1. In Experiment 1, with singlerotation sequences, the empirical probability of a response matching the simulated direction was .9 when the near-far luminance cue matched the simulated direction $[P(\operatorname{Rsim} \mid$ match $)]$; and .25 when it did not $[P(\operatorname{Rnsim} \mid$ no-match)]. The empirical probabilities of a response not matching the simulated direction for the same two conditions, $P($ Rnsim $\mid$ match $)$ and $P($ nsim $\mid$ no-match $)$, were .1 and .75 , respectively. The $P($ Reversal $)=1-$ $[P($ Rsim $\mid$ match $) \times P($ Rsim|no-match $)+P($ Rnsim $/$ match $) \times P($ Rnsim no-match). Substituting the empirical probabilities from Experiment 1 yields .71 . The computation assumes that the difference in the number of frames (three vs. seven) and the simulated sphere size $\left(4.5^{\circ}\right.$ vs. $\left.3.3^{\circ}\right)$ across experiments would not influence the estimates of the empirical probabilites significantly. There is some justification for these assumptions in that the probabilities for the first rotations in the match conditions of Experiments 2-5 were essentially the same as those of Experiment 1.

(Manuscript received August 1, 1996; revision accepted for publication February 28, 1997.) 